\title{
Escritura académica y reflexión gramatical en el comienzo de la formación universitarial ${ }^{\prime}$
}

\author{
Academic Writing and Grammatical Reflection at the Beginning \\ of University Education
}

\author{
JIMENA SCHERE \\ Consejo Nacional de Investigaciones Científicas y Técnicas (CONICET) \\ Universidad de Buenos Aires (UNAJ) \\ Universidad Nacional Arturo Jauretche (UNAJ) \\ Argentina \\ jimenaschere@hotmail.com
}

(Recibido: I8-09-20I9; aceptado: $15-03-2020)$

Resumen. El enfoque comunicativo de la lengua, implementado en la escuela argentina en la reforma de 1994, cuestionó la importancia de la gramática en la enseñanza de la lengua y privilegió nociones relativas al texto. A partir de la reforma, algunos especialistas han reclamado la necesidad de devolverle su lugar prioritario en la enseñanza de la lectoescritura. Partiendo de esta línea, entendemos que es necesario retomar los contenidos morfosintácticos, de manera instrumental y articulada con la reflexión textual y discursiva, en el inicio de la formación universitaria. Los textos producidos por muchos estudiantes iniciales revelan dificultades recurrentes a nivel de la sintaxis oracional. En este trabajo nos proponemos consignar algunos de esos errores recurrentes, relacionados frecuentemente con el uso del verbo y la sintaxis trabada propia de los textos académicos, e indagar sus posibles causas; en segundo lugar, haremos una propuesta pedagógica para abordar esta problemática en los talleres de lectoescritura académica y propiciar en los estudiantes la incorporación de la reflexión metalingüística en el proceso de escritura.

Palabras clave: escritura académica; gramática oracional; gramática textual; sintaxis trabada; reflexión metalingüística.
Abstract. The communicative approach to language, implemented in the Argentine school in the reform of 1994, questioned the importance of grammar in the teaching of language and focused on notions referring to the text. Since the reform, some specialists have claimed the need to return priority to the teaching of reading and writing. Starting from this approach, we understand that it is necessary to return to the morphosyntactic contents, in an instrumental way and articulated with the textual and discursive reflection, in the beginning of university education. The texts produced by many initial students reveal recurrent difficulties at the level of sentence syntax. In this paper we propose to record some of these recurring errors, frequently related to the use of the verb and the complex syntax of the academic texts, and investigate their possible causes; secondly, we will make a pedagogical proposal to deal with this problem in academic reading and writing classes and to promote metalinguistic reflection in the writing process.

Keywords: academic writing; initial cycle; sentence grammar; textual grammar; complex syntax; metalinguistic reflection.

\footnotetext{
${ }^{\text {I }}$ Para citar este artículo: Schere, Jimena (2020). Escritura académica y reflexión gramatical en el comienzo de la formación universitariaI. Alabe 22 . [www.revistaalabe.com]

DOI: IO.I5645/Alabe2020.22.7
} 


\section{Introducción}

El enfoque comunicativo del lenguaje, implementado en la Argentina a partir de la reforma educativa del año I994, cuestionó la importancia central de la gramática en la enseñanza de la lengua en el nivel primario y medio y, en particular, puso en duda su utilidad para el desarrollo de las capacidades de lectura y escritura de los estudiantes. El modelo comunicativo viene a sustituir el enfoque estructuralista, que privilegia el análisis sintáctico, y desplaza el estudio de la gramática oracional por la gramática textual y por el plano discursivo vinculado con el uso de la lengua (Otañi y Gaspar, 20or; Ciapuscio, 2OIO; Marín, 20I3).

A partir de esta trasformación y de las dificultades persistentes de lectoescritura observadas en los ingresantes a la universidad, algunos autores han reclamado la necesidad de devolverle a la gramática un lugar relevante en la enseñanza de la lectura y escritura y proponen articular la gramática textual con la oracional. En esta línea, Otañi (20I4) considera que los conocimientos morfosintácticos se encuentran en la base de la producción y de la comprensión de textos (Otañi, 200I; 2008). Entiende, además, que la gramática escolar, de carácter ecléctico, debe articular contenidos morfosintácticos, textuales y normativos, sujetos a una adecuada transposición didáctica (Chevallard, I99I). Con una postura semejante, Ciapuscio (2OIO) argumenta que el conocimiento gramatical interviene en la etapa de ideación y configuración conceptual de los textos y, por lo tanto, no puede limitarse a una mera función de control y revisión. Por su parte, Di Tullio (2OIO) sostiene también que la gramática contribuye de manera directa e indirecta al desarrollo de las habilidades de lectura y producción de textos.

De acuerdo con estas autoras, la reacción en la Argentina contra la gramática en los años 80 y 90 se debe a la manera inadecuada de enseñar esta disciplina, que la reduce a un ejercicio de análisis mecánico. En este sentido, Otañi, por ejemplo, propone actividades que favorezcan la reflexión gramatical en contraposición con el modelo conductista de explicación-ejercitación ${ }^{2}$.

A partir de los enfoques que plantean la importancia de la reflexión gramatical para desarrollar las capacidades de lectoescritura de los estudiantes de distintos niveles ${ }^{3}$, proponemos retomar los contenidos morfosintácticos, de manera instrumental y articulada con la reflexión discursiva, en el inicio de la formación universitaria.

Los talleres de lectoescritura académica comenzaron a implementarse en la Argentina a partir de la materia Semiología de la cátedra de Arnoux, en el pregrado de la Universidad de Buenos Aires ${ }^{4}$. Desde hace dos décadas, los talleres se han extendido en

\footnotetext{
${ }^{2}$ Por ejemplo, Giammatteo (20I3) que también considera indispensable la enseñanza de la gramática para la lectoescritura sostiene que esta disciplina se debe enseñar "a partir de los conocimientos del hablante hasta llegar reflexivamente al reconocimiento de las estructuras morfosintácticas” y propone un enfoque léxico/gramatical.

3 Un panorama actualizado sobre el debate acerca de la utilidad de la gramática escolar para el desarrollo de las habilidades de escritura presenta Tormo Guevara (20I7: 65-70).

4 Arnoux dirigió en la Cátedra de Semiología una de las primeras investigaciones sobre las prácticas de lectura y la escritura en los ingresantes a la Universidad de Buenos Aires (UBACyT FI-O3I, de I987) (Pereira, y Nogueira y Valente 20r8: I2). Los talleres del grupo de Arnoux han dado especial importancia a las aportaciones de la Teoría de la enunciación y la Lingüística textual. Véase, por ejemplo, el manual de la cátedra (Arnoux et al. 2002).
} 
los planes de estudios de las universidades argentinas, así como el trabajo investigativo en el área 5 , en el contexto de la democratización de la educación superior, del crecimiento de la matrícula de ingreso y la creación de universidades nacionales (Navarro 20I4: 86-7). En términos de Arnoux (2OI9: II), los emprendimientos en lectoescritura universitaria de las últimas dos décadas han tenido como objetivos facilitar el dominio de los géneros evaluativos habituales en los estudios superiores, la adquisición de conocimientos discursivos y de habilidades de base, el manejo de la propia lengua y la formación de un pensamiento crítico. En cuanto al lineamiento teórico, los talleres han adoptado especialmente la perspectiva del análisis del discurso (Brito, 20I6: I5). Las líneas de investigación han abordado, entre muchas otras problemáticas, la polifonía (Arnoux, Nogueira y Silvestri 200I), la reformulación (Arnoux, Nogueira y Silvestri 2006), la fragmentariedad y distorsiones enunciativas (García Negroni y Hall 20IO), la competencia argumentativa (Padilla et al. 2OIO), la enseñanza de la lectoescritura desde las formas discursivas de cada disciplina (Carlino 2005).

La importancia que tiene, sin duda, el aspecto discursivo en la enseñanza de la lectoescritura académica relega la gramática a un lugar marginal. Sin embargo, la observación de los textos producidos por los estudiantes iniciales, como exámenes y trabajos prácticos, revelan dificultades recurrentes a nivel de la sintaxis oracional y reclaman la necesidad de abordar esta problemática. En este trabajo nos proponemos, en primer lugar, consignar algunos de esos errores frecuentes, en particular, aquellos vinculados, entre otros aspectos, con el uso del verbo y con las estructuras hipotácticas y analizar sus posibles causas. En segundo lugar, haremos una propuesta pedagógica para indagar la manera en que esos errores pueden ser abordados en los talleres a partir de la reflexión gramatical. Finalmente, se intenta remarcar la necesidad de propiciar la reflexión metalingüística (discursiva, textual, gramatical) en el proceso de escritura.

\section{Errores frecuentes de escritura}

Nuestro trabajo de investigación adopta una metodología cualitativa que incluye la recolección de datos empíricos, recogidos desde el año 2014 hasta la fecha, a través de distintas herramientas, especialmente, el registro y análisis de exámenes y trabajos prácticos de estudiantes iniciales, que cursan los talleres de lectoescritura (Taller de Lectura y Escritura y/o Taller Complementario de Lengua) en su primer año de ingreso a la Universidad Nacional Arturo Jauretche, ubicada en el municipio bonaerense de Florencio Varela. Asiste a la institución un alto porcentaje de estudiantes de primera generación de universitarios proveniente de sectores populares y que, en algunos casos, presentan trayectorias escolares marcadas por la discontinuidad (Savio 2OI5: 5).

\footnotetext{
5 Para un registro y análisis contrastivo del desarrollo de talleres de lectoescritura universitaria, publicaciones del área, realización de simposios, etc. en la Argentina, véase Carlino (2006).
} 
El objetivo central de la investigación ha sido recabar errores frecuentes en el plano oracional e indagar los conocimientos morfosintácticos de los estudiantes y el empleo de la reflexión gramatical en el marco de sus prácticas concretas de escritura. Del abundante corpus de producción escrita, hemos seleccionado los trabajos que presentan problemas a nivel oracional y, entre ellos, elegimos ejemplos representativos de los casos más recurrentes. Nos centramos en la producción escrita de textos explicativos (i.e. respuesta a consigna de examen, artículo de divulgación) que los estudiantes realizan en base a un texto fuente en exámenes y trabajos prácticos de las materias de taller. Complementamos la selección y el análisis del corpus escrito con el estudio de casos, que implica el seguimiento de estudiantes con dificultades profundas a nivel oracional, a través de intercambios individuales y del registro de sus intervenciones durante las clases de taller, a fin de indagar sus conocimientos gramaticales y sus prácticas concretas de escritura.

Las producciones escritas de los estudiantes que ingresan a la universidad revelan de manera frecuente oraciones agramaticales o que no alcanzan la complejidad sintáctica propia de un texto académico, causa recurrente de desaprobación en las instancias evaluativas. Una de las dificultades más frecuentes que se presenta en el plano oracional se genera por la ausencia del verbo o por su uso incorrecto en oraciones simples, compuestas y complejas.

Es habitual que los ingresantes incluyan en sus textos oraciones sin verbo conjugado. Abundan casos de oraciones nominales o de construcciones preposicionales aisladas, por ejemplo, el siguiente enunciado escrito por un estudiante inicial: "En el texto 'La lectura y la escritura en tiempos de redes sociales'. Fabio Montero destaca la preocupación de los especialistas de la educación por la influencia de las nuevas tecnologías". El primer enunciado carente de verbo es percibido como una estructura autónoma. Asimismo, son frecuentes las oraciones nominales con verbos subordinados: "Las nuevas tecnologías que en los últimos años aumentaron en variedad como en consumo. Le (sic) ofrecen a los estudiantes una gran oportunidad de acceso a la información”.

También se observa la construcción de oraciones con núcleos verboidales, especialmente gerundios y a veces también infinitivos, en lugar de verbos conjugados. Por ejemplo, el siguiente enunciado: "Un astrónomo aficionado pudo visualizar la explosión de una estrella. Logrando así comprobar la primera fase de la teoría evolutiva de la supernova". Esta clase de construcciones proliferan en los textos de los estudiantes iniciales: "La especialista Paula Carlino sugiere ayudar a los estudiantes, antes de dar por supuesto que ya saben leer y escribir. Estando de acuerdo con las universidades estadounidenses en que leer y escribir son herramientas que abren puertas y dan poder". Además, resulta recurrente el uso incorrecto del gerundio para acción posterior y la acumulación de construcciones verboidales de gerundio en una misma oración.

Otra dificultad persistente es la tendencia a dar autonomía a proposiciones sustantivas, adverbiales y adjetivas y no reconocer la relación jerárquica entre verbos principales y subordinados. En el siguiente ejemplo se le asigna autonomía a una subordinada adverbial: "La escuela debe incorporar los formatos digitales en la enseñanza de 
la lectura y la escritura. Para que los alumnos mejoren sus producciones escritas". En este otro caso, se construye una proposición relativa adjetiva autónoma: "Víctor Buso es un astrónomo rosarino aficionado. Que en la noche logró captar el momento exacto en que estallaba una estrella”. Los ejemplos del uso de pronombres relativos autónomos se multiplican: "El texto fue publicado en la página de CONICET. El cual explica cómo un astrónomo aficionado logró captar la explosión de una estrella”.

En las proposiciones relativas es habitual también la utilización de pronombres relativos incorrectos; por ejemplo, el uso del pronombre relativo "donde", en lugar del pronombre "que" en oraciones con un sustantivo antecedente sin sentido locativo o, incluso, el uso arbitrario y autónomo del pronombre "donde" encabezando la frase: "Los estudiantes tienen dificultades para aprender a leer y a escribir. Donde se perciben errores de concordancia, puntuación y ortografía”.

Nos interesa especialmente detenernos en errores vinculados sobre todo con el reconocimiento y el uso del verbo en distintas estructuras sintácticas por su marcada recurrencia y porque generan en algunos casos profundas dificultades para la construcción de oraciones gramaticales. Sin duda, las falencias no se relacionan de manera exclusiva ni aislada con el manejo de los verbos y, por lo tanto, la problemática debe ser abordada de manera compleja ${ }^{6}$.

La construcción de oraciones sin verbo principal obedece, en parte, a la dificultad de reconocer el verbo como clase de palabra, sus características morfológicas y su función sintáctica como núcleo del predicado. Asimismo, incide la incapacidad de comprender las diferencias de uso entre una oración simple bimembre y una unimembre, y sus empleos específicos que privilegian, por ejemplo, la utilización de frases nominales para la elaboración de un título. En todos los casos es pertinente que el estudiante pueda articular los conocimientos gramaticales con los discursivos.

El trabajo personalizado de la modalidad de taller permite observar que los estudiantes que no reconocen el verbo como clase de palabra tienden a presentar errores de construcción en las oraciones de sus textos. Por ejemplo, es habitual que no puedan identificar el verbo principal de una oración simple o que confundan un sustantivo abstracto de raíz verbal como "pensamiento" con una forma verbal conjugada. Cuando se los interroga por la noción de "verbo", la respuesta más frecuente es "una acción”. Por su parte, el sustantivo suele ser definido como "una cosa”. En definitiva, las preguntas dirigidas a estudiantes con dificultades a nivel oracional revelan que por lo general cuentan con conocimientos gramaticales difusos o manejan nociones escolares simplificadas, adquiridas y fijadas en etapas tempranas de su alfabetización inicial, que implican identificar un verbo meramente con una acción. Esa visión los lleva a confundir sobre todo los verbos, los verboides y los sustantivos abstractos derivados de raíces verbales. La insistencia en las peculiaridades morfosintácticas del verbo, que lo diferencian de otras clases

\footnotetext{
${ }^{6}$ En particular, las dificultades en el uso del verbo aparecen de manera frecuente asociadas con problemas de puntuación.
} 
de palabras, resulta indispensable entonces para dotar al estudiante de un instrumento que clarifique la construcción de oraciones.

El uso del gerundio, utilizado de manera autónoma, como núcleo de la oración principal es quizás uno de los errores más habituales. Cuando se realiza la devolución de los exámenes a los estudiantes con este tipo de dificultades, se puede observar que no son capaces de identificar un verboide ni distinguirlo de un verbo conjugado. Al no reconocer las clases de palabras ni las diferencias estructurales entre una oración simple bimembre y una construcción verboidal, el estudiante carece de criterios morfosintácticos objetivos que le permitan utilizar los gerundios de manera adecuada y, también, comprender y corregir razonadamente y de forma autónoma sus textos. Las explicaciones sobre el uso correcto del gerundio ${ }^{7}$ y las correcciones de los profesores muchas veces no son asimiladas por los estudiantes, que tienden a repetir este tipo de errores por no contar con nociones gramaticales que le proporcionen una herramienta de comprensión profunda.

Finalmente, la tendencia a dar autonomía a proposiciones subordinadas (sustantivas, adverbiales, adjetivas) tiene su origen en parte también en la dificultad de reconocer los verbos y, fundamentalmente, en la ausencia de un conocimiento básico sobre las diferencias entre la oración simple, compuesta y compleja.

Resulta notable la propensión de muchos estudiantes a evitar la hipotaxis, que implica una estructura de mayor complejidad, y a poner en un mismo nivel estructuras de diferente jerarquía. Se ha observado que el fenómeno de la subordinación o sintaxis trabada es un modo de construcción que se desarrolla con posterioridad a la sintaxis suelta en el proceso diacrónico de las lenguas. La sintaxis suelta es característica de la oralidad y de formas más primarias del discurso (Badía, I96o) ${ }^{8}$. En este sentido, la tendencia observada en muchos estudiantes a ubicar en el mismo nivel estructuras de diferente jerarquía parece corresponderse con una sintaxis más primaria, cercana a la oralidad. Es necesario entonces analizar y explicitar en los talleres de lectoescritura las características propias de la sintaxis trabada que caracteriza a la escritura académica. Nuevamente, se requiere integrar el abordaje de la gramática oracional con los aspectos discursivos propios del género académico.

En los textos de estudiantes iniciales se presentan también otros errores menos frecuentes en la construcción de oraciones. Entre ellos, podemos mencionar la falta de coordinación o de puntuación entre proposiciones compuestas o entre enunciados que

\footnotetext{
7 Véanse, por ejemplo, los usos correctos e incorrectos del gerundio en García Negroni (2004: 267-270).

${ }^{8}$ A partir del análisis del Cantar de Mío Cid, Badía (1960) observa que en los comienzos de las lenguas románicas domina la yuxtaposición. El autor entiende que la sintaxis suelta, caracterizada por las construcciones yuxtapuestas, es más primaria y precede a la trabada, que comprende la subordinación. Cf. Herrero Ruz de Lóizaga (2005: 22-24). Badía también atribuye la sintaxis suelta a los individuos sin instrucción y a los niños. En las lenguas antiguas de origen indoeuropeo, también se observa el fenómeno de la parataxis. Chantraine (I953: 55I ss.) observa la predilección de la lengua épica griega por las construcciones paratácticas. Sin embargo, eso no significa que el indoeuropeo no haya conocido la subordinación (Chantraine, 1953: 232). En el indoeuropeo Rodríguez Adrados (I975: IIIO-I) entiende que las proposiciones coordinadas y subordinadas parten de oraciones simples yuxtapuestas, cuya relación no estaba explicitada. Luego las lenguas crean nexos específicos, a partir de formas prexistentes como los pronombres o adverbios anafóricos, que pasan a indicar subordinación y tipo de subordinada.
} 
podrían funcionar de manera autónoma. Estos problemas responden también al desconocimiento de la estructura de la oración simple y compuesta. Por ejemplo: "Las nuevas tecnologías generan dificultades en la lectoescritura provocan la preocupación de los especialistas". Otro caso: "La versión escolar de las prácticas sociales de lectoescritura debe ser fiel a la versión social las escuelas deben convertirse en una micro comunidad de lectores y escritores".

La falencia en los conocimientos sintácticos y la incapacidad para reconocer los verbos y su funcionamiento en la oración, provocan conjuntamente, como se puede observar en los ejemplos, errores a nivel de la puntuación, que suele ser un tema de difícil asimilación por parte de los estudiantes. Por ejemplo, entendemos que muchos de ellos transgreden la normativa que impide el uso de la coma entre sujeto y predicado porque no pueden identificar la estructura de la oración bimembre. Los problemas en el uso del punto seguido también se potencian por la dificultad de reconocer una estructura sintáctica autónoma. Enseñar el uso de la puntación es un conocimiento necesario, pero no suficiente, que debería acompañarse de la reflexión gramatical para ser comprendido en profundidad por los estudiantes.

En definitiva, observamos que los ingresantes con profundos problemas de escritura tienen conocimientos gramaticales vagos, manejan nociones excesivamente simplificadas o presentan dificultades para utilizar de manera instrumental sus conocimientos metalingüísticos en el proceso de escritura. Incluso, en algunos casos, los estudiantes mejoran sus producciones a nivel textual (rasgos estructurales del texto, progresión temática, uso de conectores, etc.), pero los problemas en el plano oracional persisten. Muchos estudiantes iniciales, por ejemplo, logran incorporar progresivamente marcadores del discurso y son capaces de organizar la estructura de sus textos (introducción al tema, desarrollo, conclusión); sin embargo, siguen produciendo oraciones sin verbo conjugado.

Lo cierto es que insistir en el reconocimiento del verbo como clase de palabra, como núcleo articulador de la oración y repasar las diferencias entre oraciones simples (bimembres, unimembres) compuestas y complejas constituye, a nuestro modo de ver, una herramienta fundamental, que si se integra con los conocimientos textuales y discursivos, puede generar mejoras en las producciones escritas de los estudiantes iniciales.

En definitiva, nuestra propuesta se enmarca en una perspectiva ecléctica que conjuga concepciones diversas: asume, en primer lugar, los postulados pedagógicos de la alfabetización académica, que propone avanzar en la enseñanza de la escritura desde la especificidad del nivel y de cada disciplina, y que reconoce asimismo las limitaciones del enfoque cognitivo y plantea la necesidad de introducir la reflexión sobre la dimensión discursiva del lenguaje (Carlino, 2004, 2005); pero además integra la línea teórica que subraya la importancia de profundizar la reflexión gramatical en los talleres, sin perder de vista las diferencias entre oración y enunciado. 


\section{Propuesta pedagógica}

Para abordar los problemas de escritura a nivel oracional, resulta pertinente, en primer lugar, realizar en los talleres una revisión general de nociones morfosintácticas, recuperando saberes previos y reviendo nociones incorporadas en los niveles educativos anteriores. El punto de inicio es el repaso de las clases de palabras, con especial insistencia en el reconocimiento del verbo conjugado y sus particularidades respecto de otras formas como los verboides. Es pertinente analizar de manera sencilla las estructuras verboidales y remarcar las diferencias morfosintácticas entre verbos conjugados y formas no personales. En segundo lugar, es preciso retomar, bajo la perspectiva de una gramática descriptiva, las diferencias entre la oración simple, compuesta y compleja de manera básica y general ${ }^{9}$. En todos los casos, resulta fundamental trabajar la diferencia entre formas verboidales y verbos conjugados y la relación jerárquica entre distintos verbos conjugados (verbo principal y verbo subordinado). El eje de trabajo es la noción de verbo y sus características morfosintácticas. Este repaso puede tomar la forma de una micro-lección, o de una serie de micro-lecciones, en términos de Weaver et. al. (2006), pero impartidas no de forma aislada, sino para integrarlas a continuación en propuestas de escritura.

Una vez trabajada la estructura oracional, resulta indispensable incorporar ejercicios para practicar las diferencias entre estas distintas formas, que propicien la reflexión sintáctica en el proceso de escritura. Una manera eficaz parece ser la corrección de textos que presenten los errores típicos en la construcción de estructuras sintácticas para que, a partir de su corrección, el estudiante ponga en práctica la indagación gramatical y advierta la utilidad de elaborar y revisar sus propias producciones con instrumentos morfosintácticos.

A modo de breve ejemplo, presento un modelo básico de ejercitación inicial que tiene en cuenta algunos de los errores típicos consignados:

\footnotetext{
9 La revisión no requiere ser exhaustiva y puede tomar como base oraciones sencillas: oración bimembre simple (p. ej. Juan estudia y trabaja), oración compuesta (p. ej. Juan estudia y Pedro trabaja), compleja (p. ej. Juan dice que quiere estudiar; Juan estudia porque quiere ser ingeniero).
} 
Los enunciados que aparecen a continuación presentan errores. Indique de qué tipo de errores se trata y proponga alternativas para corregirlos.

I. La tasa de matriculación se ha acrecentado en los últimos años tanto en las universidades públicas como en las privadas, pero también ha crecido el nivel de deserción de los estudiantes. Siendo un problema preocupante para los diversos actores sociales del área de educación.

2. La tasa de matriculación se ha acrecentado en los últimos años tanto en las universidades públicas como en las privadas, pero también ha crecido el nivel de deserción de los estudiantes. Incorporar planes de becas y tutores que acompañen a los estudiantes en el comienzo de sus carreras.

3. Los especialistas en educación manifiestan diferentes posturas sobre el problema del alto nivel de deserción de los estudiantes universitarios. Que hay que incorporar becas y tutorías en las universidades.

4. Las universidades han incorporado talleres de lectura y escritura. Para resolver las dificultades de los estudiantes que inician sus carreras universitarias.

5. Las universidades han incorporado talleres de lectura y escritura. Que tienen el objetivo de resolver las dificultades de lectoescritura de los estudiantes iniciales.

Una vez realizada la ejercitación con enunciados breves, el paso siguiente es trabajar con la corrección de textos completos más extensos, que presenten estos mismos errores y que posibilite articular la reflexión morfosintáctica, discursiva y textual. Finalmente, la propuesta debería apuntar a que los estudiantes realicen actividades de producción y de corrección de sus propios textos incluyendo en sus prácticas concretas la reflexión gramatical. Una posibilidad puede ser ejercitar la detección, corrección de errores gramaticales y la propuesta de variantes en los textos propios y/o en textos producidos por sus compañeros ${ }^{\mathrm{IO}}$.

En suma, el trabajo con el error y el esfuerzo orientado a su corrección y a la justificación de esa corrección con criterios que integren diversos planos (morfosintáctico, textal, discursivo, normativo) favorecen el desarrollo de una práctica escrituraria razonada y de una capacidad de corrección autónoma. En definitiva, remarcamos la importancia de alentar en los estudiantes la incorporación de la reflexión metalingüística en las

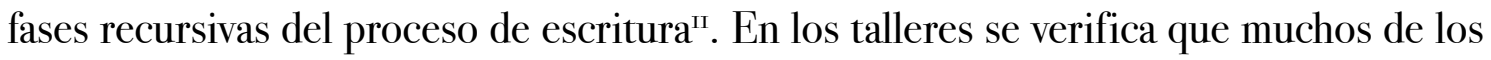

\footnotetext{
Io Muchos autores plantean que resulta más eficaz enseñar gramática en contextos de escritura que de manera aislada. Al respecto, véase por ejemplo Weaver et al. (2006); cf. Tormo Guevara (2OI7: 7I-76). Nuestra propuesta apunta a que los estudiantes puedan recuperar y afianzar nociones gramaticales con la finalidad de que sean capaces de incorporarlas de manera efectiva en sus prácticas escriturarias; de este modo, mejoran sus conocimientos metalingüísticos al tiempo que comprenden la necesidad de emplearlos en la escritura.

${ }^{\text {II }}$ Desde la psicología cognitiva, la escritura es entendida como una sucesión de procesos de planificación, traducción y revisión, de carácter recursivo y supervisados por una instancia de control (Hayes y Flower, I996).
} 
ingresantes con problemas de escritura, cuando se los interroga sobre sus prácticas concretas, manifiestan que producen sus textos en una sola fase, o bien sin ninguna o escasa planificación, sin elaboración de borradores o sin realizar correcciones sucesivas atentas a distintos aspectos del texto. Algunos también reducen al mínimo estas actividades o no les dan la suficiente relevancia en el proceso de escritura. La reflexión metalingüística propicia que el estudiante produzca sus textos en varias fases y que entienda que la escritura es un proceso complejo, alejado de la mera intuición y el automatismo ${ }^{12}$.

Por otra parte, consideramos necesario profundizar en los talleres de lectoescritura académica la dimensión conceptual de la materia, integrando nociones gramaticales, textuales y discursivas, sujetas a una adecuada transposición didáctica. La metodología de taller, indispensable en la enseñanza de la lectoescritura, genera a veces programas y cursadas de escaso desarrollo conceptual y en los propios estudiantes la falsa impresión de que no necesitan incorporar y/o implementar nociones básicas para emplearlas de manera instrumental en sus prácticas escriturarias.

La profundización de la dimensión conceptual no significa abandonar de ningún modo la metodología central de los talleres que apunta a la incorporación e implementación de esas nociones a través de ejercitación pertinente. En términos de Carlino (2015: I3), es preciso "ayudar a aprender conceptos y prácticas discursivas disciplinares por medio de tareas para reelaborar y adueñarse de unos y otros simultáneamente”.

Nuestra propuesta pedagógica se fundamenta, por último, en la constatación de que los estudiantes suelen mejorar sus producciones cuando incorporan herramientas gramaticales básicas. En cursos reducidos de estudiantes del Taller Complementario de Lengua, adonde se derivan los ingresantes con problemas más pronunciados de lectoescritura, hemos propiciado la incorporación, el repaso y la utilización instrumental de los conocimientos morfosintácticos durante el proceso de escritura, de acuerdo con los lineamientos precisados en nuestra propuesta. En algunos casos, los estudiantes incorporaron o profundizaron conocimientos que no manejaban previamente; en otros, recuperaron saberes gramaticales que, según manifestaban, habían estudiado de manera disociada de la práctica de la escritura y comenzaban a aplicarlos en el proceso de producción de textos. El dominio de herramientas gramaticales y del metalenguaje gramatical ha facilitado, en las devoluciones a los estudiantes, que pudieran comprender en profundidad las correcciones observadas en sus producciones y las razones por las que sus construcciones resultaban agramaticales o incorrectas. Además, la comprensión gramatical de esos errores permitía en muchos casos que mejoraran sus producciones a nivel oracional y que advirtieran el grado de complejidad reflexiva que implica el proceso de escritura.

\footnotetext{
I2 Rodríguez Gonzalo (20I2: IO9) presenta una serie de investigaciones didácticas que plantean la necesidad de incorporar la reflexión metalingüística explícita en los distintos niveles educativos "para posibilitar en los estudiantes el tránsito del uso espontáneo de las lenguas a los usos elaborados (orales o escritos) que la sociedad actual demanda".
} 


\section{Conclusiones}

El enfoque comunicativo en la enseñanza de la lengua ha puesto como objeto central de reflexión conceptos referidos al texto (coherencia, cohesión, tipos textuales, entre otros), indispensables para mejorar la producción escrita de los estudiantes. Sin embargo, entendemos que estos conceptos proporcionan instrumentos teóricos necesarios, pero no suficientes para solucionar problemas de escritura a nivel oracional. A partir de esta comprobación, hemos postulado que resulta necesario propiciar en el proceso de escritura la reflexión sobre sintaxis oracional, de manera complementaria y articulada con la reflexión textual y discursiva. Esta incorporación tiene como objetivo que el estudiante tenga a disposición criterios sintácticos objetivos que le permitan producir textos académicos adecuados y, también, comprender y corregir de manera autónoma los errores de escritura que produce a nivel oracional. Entendemos que es fundamental que la reflexión metalingüística no quede disociada de las prácticas escriturarias concretas de los estudiantes y que pase a formar parte activa del proceso de escritura.

A partir del análisis de los textos producidos en los talleres y de la indagación a nuestros estudiantes, observamos que muchos errores responden, al menos en parte, a la dificultad de reconocer un verbo y su uso en diferentes estructuras sintácticas. Por eso, el repaso de estas nociones y la ejercitación pertinente en textos que apunten a comprender y a subsanar este tipo de problemas, permitiría que los estudiantes mejoren sus producciones escritas. En particular, resulta necesario en los talleres detenerse en el tipo de sintaxis trabada, propia del discurso académico, y sus diferencias con la sintaxis suelta.

Una objeción frecuente en contra de la gramática han sido los resultados obtenidos en décadas pasadas que parecían demostrar que la gramática estructural no mejoraba la capacidad de lectoescritura de los estudiantes. Es evidente que la gramática no posibilita por sí sola el dominio de la escritura académica. Sin embargo, en línea con especialistas como Otañi, coincidimos en señalar que la gramática resulta una herramienta útil cuando se trasforma en un mecanismo de reflexión, se aleja del mero análisis mecánico y se integra de manera instrumental en el proceso de escritura.

En particular, en el marco específico de las universidades del conurbano a las que asisten numerosos estudiantes que pertenecen a una primera generación de universitarios, nos parece fundamental insistir especialmente en el debate. Bernard Lahire afirma que los alumnos de origen popular, a veces más distanciados de la cultura escolar, presentan dificultades para "poner el lenguaje a distancia" y tratarlo como un objeto de estudio (Bernard Lahire, 20I6: Io). Si bien la dificultad de implementar la reflexión metalingüística en el proceso de escritura parece ser generalizada, en este contexto particular, y a la vista de las serias dificultades observadas a nivel oracional, esta práctica resulta indispensable ${ }^{\mathrm{r} 3}$. Acordamos con Di Tullio (2OIO: 204), por cierto, en señalar que "entre las varias facetas de la exclusión social está la exclusión lingüística”.

I3 Teniendo en cuentan las particularidades de su alumnado, las universidades del conurbano bonaerense desarrollan estrategias de promoción más protegidas que las que rigen en la Universidad de Buenos Aires (Tiramonti, 2OII). En este sentido, la modalidad de los talleres de lectoescritura es sometida a permanente revisión. 
En definitiva, entendemos que la reflexión gramatical puede ser una herramienta más para mejorar la producción escrita de los estudiantes universitarios iniciales a nivel oracional, evitar cierto automatismo en sus prácticas escriturarias y alentar en ellos un trabajo razonado de escritura, organizado en etapas recursivas. Las razones para propiciar esta práctica en los talleres son, entre otras, la constatación de los buenos resultados observados en algunos casos con la incorporación de ejercicios de corrección de errores gramaticales típicos en textos propuestos por el docente y en las propias producciones de los estudiantes. 


\section{Referencias bibliográficas}

- Arnoux, E., Nogueira, S. y Silvestri, A. (2OOI). La escritura producida a partir de la lectura de textos polifónicos. Evaluación del desempeño de grupos con diferente entrenamiento lector previo. En Martínez, M. C. (Comp.). Aprendizaje de la argumentación razonada (pp. 49-77). Cali: Cátedra UNESCO y Universidad de Cali.

-Arnoux, E. et al. (2002), La lectura y la escritura en la universidad. Buenos Aires: Eudeba.

-Arnoux, E., Nogueira, S. y Silvestri, A. (2006). Comprensión macroestructual y reformulación resuntiva de textos teóricos en estudiantes de institutos de formación de docentes primarios. Signos, 39 ,6o, 9-30.

-Arnoux, E. (2019). "Prólogo". En Glozman, M. y Savio, K. Manual para estudiar textos académicos. Prácticas, conceptos y métodos en los procesos de lectura y escritura (pp. II-I3). Buenos Aires: Noveduc.

- Badía Margarit, A. M. (I96o). Dos tipos de lengua, cara a cara. En AAVV. Studia Philologica: homenaje ofrecido a Dámaso Alonso por sus amigos y discípulos con ocasión de su 60 aniversario, T. I (pp. II5-39). Madrid: Gredos.

- Brito, A. (20i6). Clase 9. Leer y escribir en la universidad. Especialización en Lectura, Escritura y Educación (Cohorte Io). Obtenido en octubre de 2016 desde Flacso http://virtual. flacso.org.ar/mod/book/view.php?id=I2326I

- Carlino, P. (2004). (Comp.). Leer y escribir en la universidad. Buenos Aires: Lectura y Vida.

- Carlino, P. (2005). Escribir, leer y aprender en la universidad. Una introducción a la alfabetización académica. Buenos Aires: Fondo de Cultura Económica.

- Carlino, P. (2006). Concepciones y formas de enseñar escritura académica. Un estudio contrastivo. Signo \& Seña, I6, 7 ${ }^{\mathrm{I}-\mathrm{II}} 7$.

- Chantraine, P. (i953). Grammaire homérique. T. II: Syntaxe. Paris: Klincksieck.

- Chevallard, Y. (199I). La transposición didáctica. Del saber sabio al saber enseñado. Buenos Aires: Aique.

- Ciapuscio, G. (2OIO). Revisión crítica y propuesta para la enseñanza de la gramática en la escuela primaria En AAVV. La formación docente en alfabetización inicial 2009 - 2010 (pp. I83-199). Buenos Aires: Instituto Nacional de Formación Docente, Ministerio de Educación. 
- Di Tullio, Á. (2OIO). Reflexiones sobre el lugar de la gramática en la escuela primaria. En AAVV. La formación docente en alfabetización inicial 2009 - 2010 (pp. 2OI-2I3). Buenos Aires: Instituto Nacional de Formación Docente, Ministerio de Educación.

- Flower, L. y Hayes, J. (I996 [I981]). La teoría de la redacción como proceso cognitivo. En Textos en contexto. Los procesos de lectura y escritura (pp. 73-IIо). Buenos Aires: Lectura y vida.

- García Negroni, M. M. (2004) (Coord.). El arte de escribir bien en español. Manual de corrección de estilo. Buenos Aires: Biblos.

- García Negroni, M. M. y Hall, B. (2OIO). Escritura universitaria, fragmentariedad y distorsiones enunciativas. Propuestas de prácticas de lectura y escritura focalizadas en la materialidad lingüístico-discursiva. Boletín de Lingüística XXII, 34, 4I-69.

- Giammatteo, M. (20I3) • ¿Por qué y para qué enseñar gramática? La gramática en la formación de habilidades cognitivo-lingüísticas. SIGNOS ELE, 7. Obtenido en diciembre de 2018 de http://p3.usal.edu.ar/index.php/ele/article/view/2003.

- Herrero Ruiz de Lóizaga, F. J. (2005). Sintaxis histórica de la oración compuesta en español. Madrid: Gredos.

- Lahire, B. (20i6). Clase i: Los orígenes de la desigualdad escolar: cultura escrita escolar, lecturas populares y configuraciones familiares. Especialización en Lectura, Escritura y Educación (Cohorte Io). Obtenido en octubre de $20 \mathrm{O} 6$ desde Flacso http://virtual.flacso. org.ar/mod/book/view.php?id=I23I5I

• Marín, M. (20I3 [1999]). Lingüística y enseñanza de la lengua. Buenos Aires: Aique.

- Navarro, F. (20I4). Manual de escritura para carreras de humanidades. Buenos Aires: Editorial de la Facultad de Filosofía y Letras.

- Otañi, L. y Gaspar, Ma. (200I). Sobre la gramática. En Alvarado, M. (Comp.). Entre líneas. Teorías y enfoques en la enseñanza de la escritura, la gramática y la literatura (pp. 75-III). Buenos Aires: FLACSO / Manantial.

- Otañi, L. (2008). Una gramática reflexiva y contextualizada. Limen, 8, 22-25.

- Otañi, L. (20I4). Clase 27: La enseñanza de la gramática. Diploma Superior en Lectura, Escritura y Educación (Cohorte Io). Obtenido en septiembre de 2016 desde Flacsohttp:// virtual.flacso.org.ar/mod/book/view.php?id=I4235.

- Padilla, C., Douglas, S. y López, E. (2OIO). Competencias argumentativas en la alfabetización académica, Revista Innovación Educativa, 4. 
- Pereira, M. C., Nogueira S. y Valente, E. (2018). “Introducción”. En Bein, R., Bonnin, J. E., di Stefano, M., Lauria, D. y Pereira, M. C. (Coord.). Homenaje a Elvira Arnoux. Estudios de análisis del discurso, glotopolítica y pedagogía de la lectura y la escritura. Tomo III: Lectura y escritura (pp. II-I5). Buenos Aires: Editorial de la Facultad de Filosofía y Letras.

• Rodríguez Adrados, F. (1975). Lingüistica indoeuropea. Madrid: Gredos.

- Rodríguez Gonzalo, C. (20I2). La enseñanza de la gramática: las relaciones entre la reflexión y el uso lingüístico. Revista ibero-americana de educación, 59, 87-ı18.

- Savio, K. (2OI5). La lectura y la escritura: un estudio sobre representaciones sociales de estudiantes universitarios. Revista Electrónica "Actualidades Investigativas en Educación", I5/2, I-26.

- Tiramonti, G. (2OII). Educación secundaria argentina: dinámicas de selección y diferenciación. Cuadernos de Pesquisa, 4I/I44, 692-705.

-Tormo Guevara, E. (20I7). Reflexión metalingüística en el proceso de composición escrita cooperativa en el marco de una secuencia didáctica. Tesis doctoral. Barcelona: Universitat Autònoma de Barcelona. Departament de Didàctica de la Lengua, de la Literatura i de les Ciències Socials.

- Weaver, C., Bush, J., Anderson, J., y Bills, P. (2006). Grammar intertwined throughout the writing process: An "inch wide and a mile deep", English Teaching, 5/, 77-IOI.

Una versión preliminar de este trabajo ha sido presentada en el II Congreso Nacional Cátedra UNESCO para la lectura y la escritura, realizado en Mar del Plata, Buenos Aires, en abril de 20I9. 